\title{
SULLE ONDE SEMPLICI DI TIPO PERMANENTE E ROTAZIONALE ${ }^{1}$ ).
}

Nota del Prof. UMBERTo CISOTTI.

Si abbia un liquido pesante in equilibrio, in un canale rettilineo indefinito, a fondo orizzontale e a sponde verticali, parallele tra di loro.

Si immagini di turbare abbastanza poco l' equilibrio del liquido, di guisa che il pelo libero, prima perfettamente liscio, divenga ora loggermente increspato. Ciò si può ottenere, per es., mediante un opportuno getto istantaneo di aria sullo specchio libero. Si propaga allora nel canale un movimento ondulatorio che è stato oggetto di ricerche classiche.

Ordinariamente si ammette che il movimento abbia luogo per piani paralleli alle sponde, e sia irrotazionale. Ed ì notoria la esistenza di una soluzione approssimata delle equazioni idrodinamiche, corrispondente a onde progressive semplici, di profilo sinusoidale ${ }^{2}$ ). In questa soluzione, la velocità $c$ di propagazione dell' onda si esprime in funzione della sua lunghezza $\lambda$ e della profondità media $h$ [rapporto tra la portata apparente ${ }^{3}$ ) $q$ e la velocità $\left.c\right]$ mediante la relazione di Airy:

$$
c^{2}=\frac{g \lambda}{2 \pi} \operatorname{tgh} \frac{2 \pi h}{\lambda},
$$

$g$ essendo l'accelerazione di gravità.

Non mi sembra privo di interesse saggiare la esistenza, nello stesso ordine di approssimazione, di onde progressive

1) R. Istituto Lombardo di S'cienze e Lettere. Vol. XLVI, fasc. 16-17.

3) Cfr. ad es. Lamb. "Lehrbuch der Hydrodynamik ", (trad. tedesea). [Teubner, 1907, §§ 228.230].

$\left.{ }^{3}\right)$ Cioè rispetto ad un osservatore che accompagna l'onda. 
semplici, aventi ancora profilo sinussoidale, ma più generali di quelle studiate da Airy.

Ciò forma argomento della presente Nota.

Abbandonando l'ipotesi della irrotazionalità del moto ondoso, si trova appunto che esistono infiniti tipi di onde progressive sinussoidali, aventi carattere permanente e ratazionale

Per tutti il vortice risulta costante sia sopra ogni orizzontale, sia (a meno di quantità di ordine superiore) sul pelo libero. Si constata che

$$
h=\frac{q}{c},
$$

non rappresenta più, come nel caso irrotazionale, la profondità media del canale, ciò̀ il valor medio delle altezze del pelo libero sul fondo, ma questa $-\mathrm{H}-\dot{e}$ invece legata a quella dalla relazione:

$$
\mathrm{H}=h+v f(h),
$$

essendo $f$ una funzione arbitraria che caratterizza la distribuzione dei vortici e $\vee$ una costante infinitesima, pure arbitraria, che ne regola la intensità.

$\dot{\mathrm{E}}$ pertanto opportuno di chiamare: $h$ profondità media fittizia; $\mathrm{H}$ profondità media reale. Esse coincidono per le onde irrotazionali $(\nu=0)$.

E degna di rilievo la circostanza che continuano a valere tutti $i$ risultati relativi alle onde irrotazionali, e in particolare la relazione (I), purchè $h$ venga ovunque sostituita con $H$. Che se si vuole introdurre in (I), a mezzo di (III), la profondità media fittizia $h$, si ha la formula di Airy generalizzata :

$$
c^{2}=\frac{g \lambda}{2 \pi} \operatorname{tgh} \frac{2 \pi h}{\lambda}+g \vee f(h) \operatorname{sech}^{2} \frac{2 \pi h}{\lambda} .
$$




\section{$\S 1$.}

Equazioni indefinite ridotte alla prima approssimazione.

Sieno: $u^{*}$ la componente orizzontale (nel senso della propagazione dell'onda) della velocità in un punto $\mathrm{P}, v^{*}$ la componente verticale, ascendente.

Posto

$$
\left\{\begin{array}{l}
u^{*}=c u \\
v^{*}=c v
\end{array}\right.
$$

dove $c$ è il valore assoluto della velocità di propagazione dell'onda, le quantità

$$
u=\frac{u^{*}}{c}, \quad v=\frac{v^{*}}{c},
$$

sono puri numeri, inquantochè entrambi sono rapporti di velocità.

Ammetterò che $u$ e $v$ e le loro derivate sieno quantità di primo ordine, con che si viene a tradurre in forma analitica la circostanza che la perturbazione del liquido è piccola.

Sia $O x y$ una coppia di assi, dotata della traslazione apparente della massa liquida, coll' asse $\mathrm{O} x$ coincidente col fondo del canale e diretto nel senso opposto alla traslazione e l'asse $O y$ verticale ascendente. La velocità di propagazione del moto ondoso ha per componenti - $c, o$.

Sieno ancora: $g$ il valore assoluto dell' accelerazione di gravità; $p$ il valore assoluto della pressione specifica in un punto generico.

Se si assume $==1$ la densità costante del liquido, le equa. zioni indefinite che legano $u^{*}=c u, v^{*}=c v, p, g$ sono notoriamente, a meno di quantità di ordine superiore al primo:

$$
\left\{\begin{array}{c}
c \frac{\partial u}{\partial t}=-\frac{\partial p}{\partial x}, \\
c \frac{\partial v}{\partial t}=-\frac{\partial p}{\partial y}-g, \\
\frac{\partial u}{\partial x}+\frac{\partial v}{\partial y}=0 .
\end{array}\right.
$$


Se si ammette che il moto perturbato abbia carattere permanente, rispetto agli assi mobili, $\dot{e}$

$$
\begin{aligned}
& \frac{\partial u}{\partial t}=c \frac{\partial u}{\partial x} \\
& \frac{\partial v}{\partial t}=c \frac{\partial v}{\partial x} .
\end{aligned}
$$

Ciò posto le equazioni (2) divengono in definitiva:

(3)

$$
\left\{\begin{array}{c}
c^{2} \frac{\partial u}{\partial x}=-\frac{\partial p}{\partial x} \\
c^{2} \frac{\partial v}{\partial x}=-\frac{\partial p}{\partial y}-g \\
\frac{\partial u}{\partial x}+\frac{\partial v}{\partial y}=0
\end{array}\right.
$$

$\S 2$.

\section{Riduzione del sistema (3). - Integrale generale.}

$\mathrm{Si}$ soddisfa, nel modo più generale, all'ultima delle equazioni (3), introducendo una funzione $\psi(x, y)$, e ponendo

$$
\left\{\begin{array}{l}
u=\frac{\partial \Psi}{\partial y}, \\
v=-\frac{\partial \Psi}{\partial x} .
\end{array}\right.
$$

Se $f^{\prime}(y)$ designa una funzion̨e arbitraria della sola $y$, e si indicano con $f^{\prime}(y)$ e $f^{\prime \prime \prime}(y)$ le sue derivate, prima e seconda, la eliminazione della pressione $p$ tra le due prime di (3), porge l' equazione

$$
\Delta \psi=\nu f^{\prime \prime}(y),
$$

cui deve soddisfare la funzione $\psi(x, y)$. Con $\vee$ indico una costante arbitraria infinitesima, la quale serve a mettere in rilievo che la funzione $\psi$ e le sue derivate vanno trattate come quantità di primo ordine. 
Il sistema (3) risulta pertanto equivalente a quello costituito dalle equazioni (4), (5) e dalla seguente:

$$
p=c^{2}\left\{-\frac{\partial \psi}{\partial y}+v f^{\prime}(y)\right\}-g y+\text { costante }
$$

la quale definisce in ogni punto la pressione $p$, una volta che sia nota la funzione $\psi$ e sia assegnata la $f$.

A norma di (1) $\odot$ (4) la funzione di corrente di Stokes $\dot{\theta}$

$$
\psi^{*}=c \psi
$$

Ne segue, che le linee di corrente assolute hanno per equazioni

$$
\psi=\text { costante }
$$

mentre lo linee di corrente apparenti (quali risultano cioè ad un osservatore dotato del moto degli assi $\mathrm{O} x y$ ) hanno le equazioni

$$
y+\psi=\text { costante . }
$$

L' integrale generale di $(5) \grave{\theta}$

$$
\psi(x, y)=\alpha \mathrm{A}(x, y)+\nu f(y),
$$

dove $\mathbf{A}(x, y)$ rappresenta una funzione armonica arbitraria, ed $\alpha \grave{\theta}$.una sostante arbitraria infinitesima.

Facendo in (9) $\alpha=0$, si ha la soluzione particolare

$$
\psi=\nu f(y) \text {. }
$$

Da questa, tenuto conto di (8), scende che il moto ad essa corrispondente da luogo a linee di flusso apparenti che sono rette orrizzontali, parallele al fondo del canale. Inoltre per la (10) stessa, la (6) diviene

$$
p=-g y+\text { costante }
$$

ciò̀ la pressione idrodin tmica coincide colla pressione idrostatica ${ }^{4}$.

1) Cfr. Cisotti. a Moti di un liquido che lasciano inalterata la distribuzione locale delle pressioni $*$ [Rend. della R. Accademia dei Lincei. Serie V, Vol. XIX, pag. 373-376]. 
Il vortice

$$
\omega=\frac{c}{2}\left(\frac{\partial v}{\partial x}-\frac{\partial u}{\partial y}\right)=-\frac{c}{2} \Delta_{2} \psi=-\frac{c}{2} f^{\prime \prime}(y)
$$

che è perpendicolare al piano del moto, ha valore costante sopra ogni linea di flusso.

\section{$\S 3$.}

\section{Le onde irrotazionali di Airy. - Condizioni ai limiti.}

Se si tratta, in particolare, di onde irrotazionali ̀̀

$$
v=0 \text {, }
$$

e si pud, secondo Airy, attribuire a $\psi$ l' espressione '):

$$
\psi(x, y)=\alpha \mathrm{A}=2 \alpha \operatorname{senh} \frac{2 \pi y}{\lambda} \cos \left(\frac{2 \pi x}{\lambda}+\varphi\right)
$$

la costante (infinitesima) $\alpha$, a priori arbitraria, dipende dal. l'altezza dell' onda, $\beta$ è una costante arbitraria e $\lambda$ è la lunghezza d'onda.

La (13) soddisfa alla condizione relativa al fondo del canale :

$$
\psi=0 \text { per } y=0
$$

e soddisfa inoltre alla condizione che riguarda il pelo libero:

$$
p=\text { costante per } c(\psi+y)=q,
$$

- q portata apparente della corrente - purchè, com'è ben noto, la velocità di propagazione c dell' onda sia legata alla sua lunghezza $\lambda$ ed alla profondità media del canale

$$
h=\frac{q}{c},
$$

dalla relazione di Airy:

$$
c^{2}=\frac{g \lambda}{2 \pi} \operatorname{tgh} \frac{2 \pi h}{\lambda}
$$

1) Cfr Lamb., l. c. $\$ 230$. 
$\S 4$.

\section{Le onde rotazionali.}

Considero ora il moto più generale, definito dalla (8)

$$
\psi(x, y)=\alpha \mathrm{A}(x, y)+\nu f(y),
$$

dove $\mathrm{A}(x, y)$ ha l' espressione che risulta da (13).

Se si impone alla $f(y)$ di annullarsi per $y=0$, cirè che sia

$$
f(o)=0,
$$

rimane soddisfatta la condizione (14) relativa al fondo del canale.

Cerchiamo ora di rendere soddisfatta la condizione (15) che si riferisce al pelo libero.

Converrà a tal uopo avere presenti le espressioni che assumono, nel caso attuale, la pressione $p$ e la funzione di corrente $\psi^{*}$.

Da (6) per (9) e (13) si ha:

(19) $p=-\frac{4 \pi \alpha c^{2}}{\lambda} \cosh \frac{2 \pi y}{\lambda} \cos \left(\frac{2 \pi x}{\lambda}+p\right)-g y+$ costante mentre ora

$$
\psi^{\prime}=e 2 \alpha \operatorname{senh} \frac{2 \pi y}{\lambda} \cos \left(\frac{2 \pi x}{\lambda}+i\right)+\nu f(y) .
$$

Sia $y=I I$ il livello medio; posto

$$
y=\mathrm{H}(1+\eta),
$$

$\eta \mathrm{H}$ rappresenta, in ogni punto, il sopraelevamento [positivo verso l'alto, negativo verso il basso] del liquido dal livollo medio, e quindi :

$$
\eta=\frac{\eta \mathrm{H}}{\mathrm{H}},
$$

è il rapporto fra detto sopraelevamento ed il livello medio $\mathrm{H}$. Come si vede $\eta$ è un puro numero, in quanto è rapporto di due lunghezze. 
Sopra il pelo libero $\eta$ è a ritenersi quantità di primo ordine. Si avrà pertanto, ivi:

$$
\begin{gathered}
\cosh \frac{2 \pi y}{\lambda}=\cosh \frac{2 \pi \mathrm{H}}{\lambda}+2 \pi \eta \frac{\mathrm{H}}{\lambda} \operatorname{senh} \frac{2 \pi \mathrm{H}}{\lambda}, \\
\operatorname{senh} \frac{2 \pi y}{\lambda}=\operatorname{senh} \frac{2 \pi \mathrm{H}}{\lambda}+2 \pi \eta \frac{\mathrm{H}}{\lambda} \operatorname{sosh} \frac{2 \pi \mathrm{H}}{\lambda}, \\
f(y)=f(\mathrm{H})+\eta \mathrm{H} f^{\prime}(\mathrm{H}) .
\end{gathered}
$$

Per queste possiamo ricavare da (19) e (20) le espressioni che spettano a $p$ e a $\psi^{*}$ rispettivamente sul pelo libero.

Si ottiene, a meno di quantità di ordine superiore:

(19) $p=-\frac{4 \pi \alpha c^{2}}{\lambda} \cosh \frac{2 \pi \mathrm{H}}{\lambda} \cos \left(\frac{2 \pi x}{\lambda}+\rho\right)-g y+$ costante;

(20) $\quad \psi=e 2 \alpha \operatorname{senh} \frac{2 \pi \mathrm{H}}{\lambda} \cos \left(\frac{2 \pi x}{\lambda}+\beta\right)+v f(\mathrm{H})$.

Ciò posto, si vede immediatamente che la condizione (15), relativa al pelo libero, è soddisfatta ponendo:

$$
c^{2}=\frac{g \lambda}{2 \pi} \operatorname{tgh} \frac{2 \pi \mathrm{H}}{\lambda},
$$

mentre il pelo libero é, come nelle onde irrotazionali, sinussoidale, ed ha per equazione:

$$
y=\frac{q}{c}-2 \alpha \operatorname{senh} \frac{2 \pi \mathrm{H}}{\lambda} \cos \left(\frac{2 \pi x}{\lambda}+\beta\right)+\nu f(\mathrm{H}) .
$$

Posto, come in (16),

$$
h=\frac{q}{c},
$$

da (23) si ricava per la profondità media $H$, l'espressione definita dalla relazione funzionale:

$$
\mathbf{H}=\frac{1}{\lambda} \int_{0}^{\lambda} y d x=h+v f(\mathbf{H})
$$


La parte funzionale può farsi scomparire se si nota che risultando da (24) $\mathrm{H}$ somma di un termine finito $[h]$ e di un termine di primo ordine $[v f(\mathrm{H})] \dot{e}$ - a meno di quantità di ordine superiore al primo -

$$
\vee f(\mathrm{H})=v f(h)
$$

allora alla (24) si può sostituire la seguente :

$$
\mathrm{H}=h+v f(h) \text {, }
$$

$h$ - rapporto tra la portata apparente e la velocità di pro. pagazione dell' onda - può chiamarsi profondità media fitti. zia; H - valor medio delle altezze del pelo libero sul fondo - è a dirsi profondità media reale. Da (25) risulta che, in generale, esse sono distinte; coincidono per le onde irrotazionali $[\nu=0]$.

Per la (25), l'equazione (23) del pelo libero può scriversi :

$$
y=H-2 \alpha \operatorname{senh} \frac{2 \pi H}{\lambda} \cos \left(\frac{2 \pi x}{\lambda}+\beta\right) \text {. }
$$

Concludendo: alle onde rotazionali si possono applicare tutti $i$ risultati relativi a quelle irrotazionali, purchè $h$ venga sostituito con $\mathrm{H}$.

La formula (22) esprime la velocità di propagazione del. l'onda mediante la profondità media reale $\mathrm{H}$. Si può, a mezzo di (25), introdurre la profondità media fittizia $h$ ed ottenere cosi la formula di Airy generalizzata.

Essendo, infatti, colla solita approssimazione:

$$
\operatorname{tgh} \frac{2 \pi G}{\lambda}=\operatorname{tgh} \frac{2 \pi h}{\lambda}+v \frac{2 \pi f(h)}{\lambda} \operatorname{sech}^{2} \frac{2 \pi h}{\lambda},
$$

la (22) può scriversi :

$$
c^{2}=\frac{g \lambda}{2 \pi} \operatorname{tgh} \frac{2 \pi h}{\lambda}+\nu g f(h) \operatorname{sech}^{2} \frac{2 \pi h}{\lambda},
$$

che è appunto la formula annunciata. 